\title{
Wide band UV/Vis/NIR blazed-binary reflective gratings: two lithographic techniques investigation
}

\author{
Mane-Si Laure Lee ${ }^{1}$, Julie Cholet ${ }^{1}$, Anne Delboulbé ${ }^{1}$, Raphaël Guillemet ${ }^{1}$, Brigitte Loiseaux ${ }^{1}$, Patrick Garabedian ${ }^{1}$, \\ Thomas Flügel-Paul ${ }^{2}$, Tino Benkenstein ${ }^{2}$, Susann Sadlowski ${ }^{2}$, Nicolas Tetaz ${ }^{3}$, Roman Windpassinger ${ }^{4}$, Saroj Mahalik \\ ${ }^{1}$ Thales Research \& Technology, 1 Av Fresnel, 91767 Palaiseau, France \\ ${ }^{2}$ Fraunhofer Institute for Applied Optics and Precision Engineering, Albert-Einstein-Strasse 7, 07745 Jena, Germany \\ ${ }^{3}$ Thales Alenia Space, 5 Allée des Gabians, BP 99, 06156 Cannes la Bocca Cedex, France \\ ${ }^{4}$ European Space Research and Technology Centre, Postbus 299-2200 AG Noordwijk, The Netherlands
}

\begin{abstract}
We report on subwavelength reflective gratings for hyperspectral applications operating in the $340 \mathrm{~nm}-1040 \mathrm{~nm}$ spectral range. The blazed grating period is $30 \mu \mathrm{m}$ and is composed of 2D subwavelength binary structures with sizes in-between $120 \mathrm{~nm}$ and $350 \mathrm{~nm}$. We demonstrate the manufacturing of gratings on 3" wafers by two lithography technologies (e-beam or nanoimprint) followed by dry etching process. These subwavelength gratings enable broadband efficiency which is in average $15 \%-20 \%$ above the efficiency requirement for next generation of spectro-imagers for Earth observation missions and a wavefront error that is much smaller than the $100 \mathrm{~nm}$ requirement for space application.
\end{abstract}

\section{Introduction}

Next generation of spectro-imagers for future Earth observation missions requests ultra-wide band operation, very large field of view but small volume. However, the performance of these future instruments is limited by the performance of the diffraction grating, since high diffraction efficiency and reduced polarization sensitivity over wide spectral range are required, while introducing a limited wavefront error. In this paper, we report on gratings with period of $30 \mu \mathrm{m}$ and operating at an angle of incidence of $0.5^{\circ}$, matching the specific requirements of a Dyson spectrometer [1]. The required minimum throughput lies only between $35 \%$ and $50 \%$ but must be achieved within $340 \mathrm{~nm}-1040 \mathrm{~nm}$ spectral range.

Classical continuous reflective saw-tooth profile blazedgratings offer peak efficiencies up to $\sim 90 \%$ (mainly limited by the reflectivity of the metallic material) but it decreases significantly when approaching the band edges in the UV and NIR regions. To overcome this limited spectral efficiency, several approaches have been proposed in the literature. The first approach consists in combining several materials to achromatize the efficiency. Originally proposed for transmission gratings $[2,3]$, it has been applied recently to reflection gratings [4]. Another alternative is the so-called "multi-blaze" or "multi-angle" approach [5, 6]. Different sawtooth depths or different facet angles are combined within the same grating.
A third type of approach is based on blazed-binary gratings composed of subwavelength structures [7, 8] and effective index dispersion engineering. Recently, we reported on the realization of a subwavelength grating by e-beam lithography and etching [9].

Here, we evaluate two lithography techniques: e-beam and NanoImprint Lithography (NIL) and compare their performance with next generation spectro-imager requirements, which are summarized in Table 1. NIL is a replication technology enabling cost-effective manufacturing process fitted for low/medium-volume for earth observation missions.

Table 1. Grating requirements related to UV/Vis/NIR spectro-imager.

\begin{tabular}{|c|c|}
\hline Spectral band & $340 \mathrm{~nm}-1040 \mathrm{~nm}$ \\
\hline $\begin{array}{l}\text { Diffraction efficiency in } \\
\text { the -1st diffraction order }\end{array}$ & $\begin{array}{l}=340 \mathrm{~nm}:>0.5 \\
=440 \mathrm{~nm}:>0.4 \\
=800 \mathrm{~nm}:>0.35 \\
=1040 \mathrm{~nm}:>0.35\end{array}$ \\
\hline Polarization sensitivity & $<10 \%($ goal $<1 \%)$ \\
\hline $\begin{array}{l}\text { Spectral ghosts } \\
\text { intensities }\end{array}$ & $\begin{array}{l}<1 \mathrm{e}-4 \text { (with respect to useful } \\
\text { diffracted order) }\end{array}$ \\
\hline Wavefront error & WFE < $100 \mathrm{~nm}$ RMS \\
\hline
\end{tabular}




\section{Subwavelength gratings fabrication}

The design of the blazed-binary subwavelength grating is based on artificial material engineering [10] and purposeful design of size, shape and arrangement of the sub-wavelength features for tailoring the grating's spectral characteristics. The $30 \mu \mathrm{m}$ grating period is composed of $2 \mathrm{D}$ subwavelength binary structures with sizes from $120 \mathrm{~nm}$ to $350 \mathrm{~nm}$ and depth of $\sim 510 \mathrm{~nm}$.

For gratings realization, one main process flow, depicted in Fig.1, was followed in two locations: Institute Fraunhofer for Applied Optics and Precision Engineering (IOF) and Thales Research \& Technology (TRT).

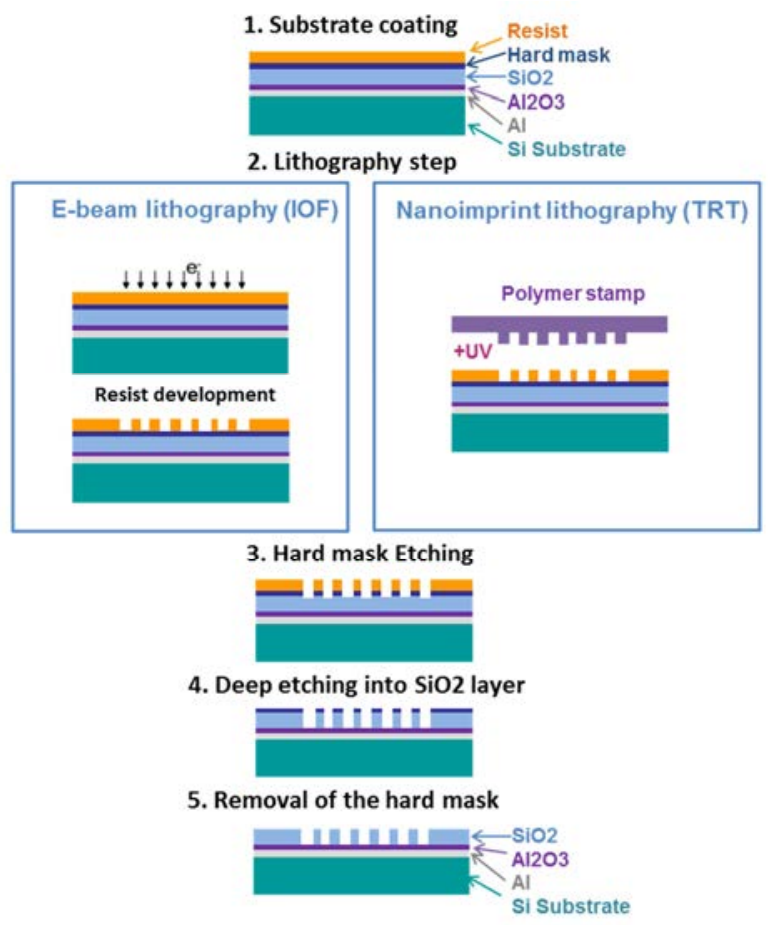

Fig. 1. Process flow for the realization of the gratings.

We use a lithography step to define the patterns into a resist layer, followed by dry etching processes to i) transfer the patterns into the metal mask and ii) to transfer the patterns into the underlying $\mathrm{SiO}_{2}$ material and get high aspect ratio structures.

Regarding the lithography step, two techniques were investigated: e-beam lithography (IOF), which is the most common technique to tackle such small size patterns, and NIL (TRT). The NIL is a replication technology which consists in fabricating a transparent polymer stamp (polydimethylsiloxane PDMS) and applying the stamp onto a photosensitive resist layer cured under UV light. The polymer stamp which is illustrated in Fig. 2, is previously produced from a flat silicon master mold fabricated with e-beam lithography (at IOF). Thanks to a Surface Conformal Imprint Lithography module from Süss MicroTec, one stamp enables several replications with a high reproducibility and homogeneous patterning over 3" surface.
The advantage of NIL enables the same high resolution as e-beam lithography, with reduced process time (typically few minutes per wafer vs few hours). Pictures of two gratings are given in Fig. 3.
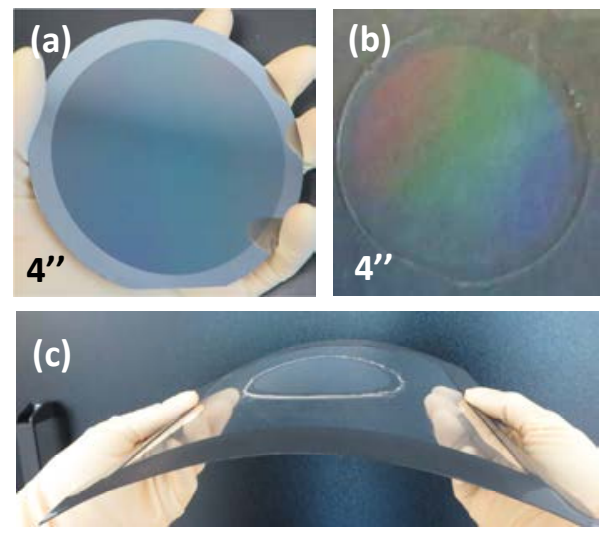

Fig. 2. Silicon master (4") (a) and manufactured PDMS mold (b) fixed on a thin glass plate (c) used for the NIL grating manufacturing.

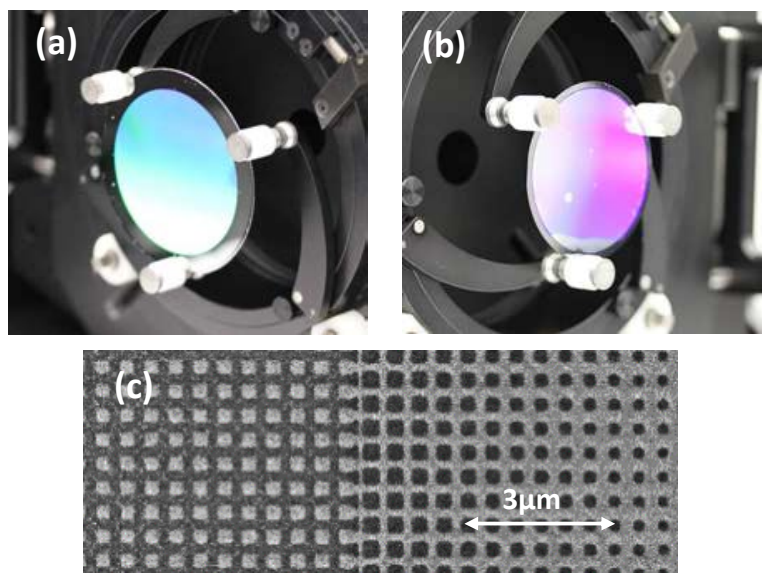

Fig. 3. Manufactured gratings on 4" wafer by e-beam lithography (a) and on 3" wafer by NIL (b). (c) SEM top view of few subwavelength structures of the NIL grating.

\section{Optical measurements}

The $\sim 80 \mathrm{~mm}$ diameter blazed-binary subwavelength gratings were characterized optically at IOF premises.

The efficiency measurements were performed using a tunable light source. The gratings are illuminated by a monochromatic light beam (diameter: $\sim 3 \mathrm{~mm}$ ) with adaptable wavelength (FWHM $<5 \mathrm{~nm})$. Measurements are carried out under TE and TM polarization. The diffracted light is detected using an integrating sphere which is mounted on a goniometer setup.

Fig. 4 shows efficiency maps at $376 \mathrm{~nm}, 600 \mathrm{~nm}$ and $1000 \mathrm{~nm}$ for the subwavelength grating by e-beam lithography. The mapping sampling is $4 \mathrm{~mm} \times 4 \mathrm{~mm}$. It shows the large-scale uniformity of the manufactured grating. 


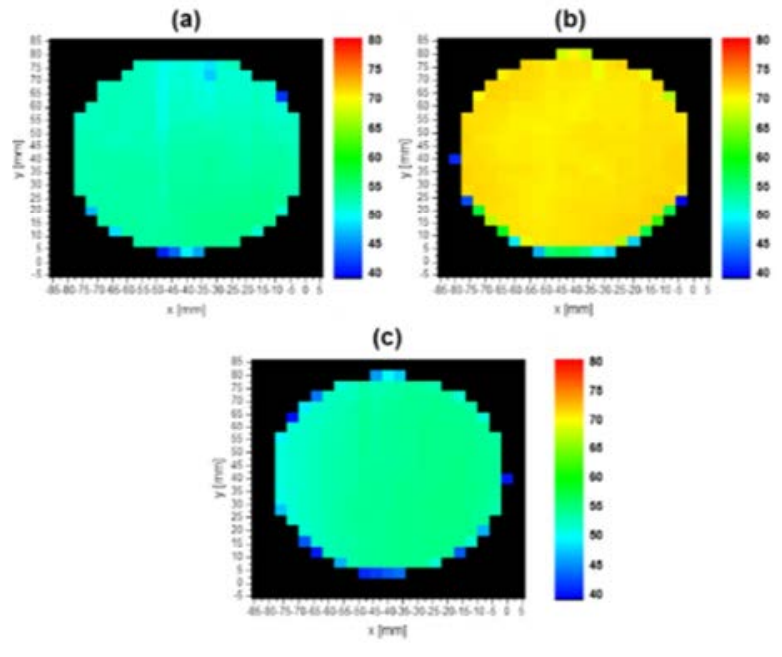

Fig. 4. Spatial mapping of the diffraction efficiency in $-1^{\text {st }}$ order of the prototype flat grating for three different wavelengths, i.e. $376 \mathrm{~nm}$ (a), $600 \mathrm{~nm}$ (b) and $1000 \mathrm{~nm}$ (c).

Fig. 5 illustrates the $-1^{\text {st }}$ order reflective efficiency of the gratings illuminated under unpolarized light and at $1^{\circ}$ angle of incidence. The black line corresponds to the ebeam grating, as compared to the green line (design efficiency), and the red line (requirement). The blue line corresponds to the NIL grating with a $50 \mathrm{~nm}$ depth deviation with respect to the nominal depth.

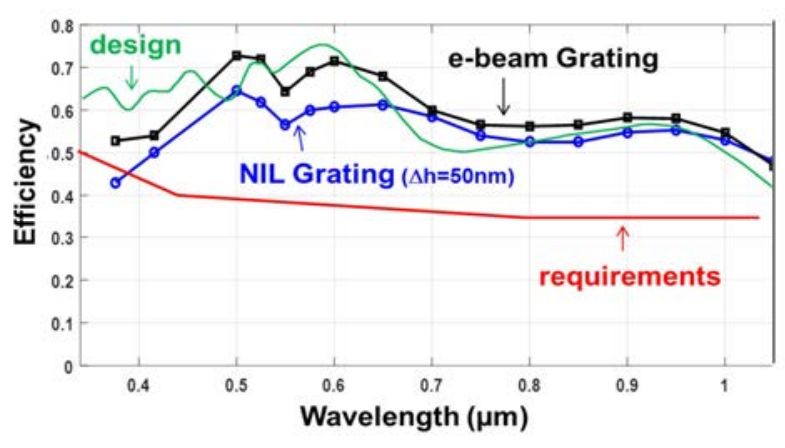

Fig. 5. Measured $-1^{\text {st }}$ order diffraction efficiency of blazedbinary subwavelength gratings.

From Fig. 5, two main results are straightforward.

- First, both e-beam and NIL give similar efficiency behavior which is in average $10 \%$ $20 \%$ above the requirement (red line).

- Secondly, the proposed design (green line) and sub-wavelength grating technology (black and blue line) are tolerant to height deviation of $50 \mathrm{~nm}$ (blue line) and efficiency remains compatible with requirements.

Fig. 6 illustrates the $-1^{\text {st }}$ order reflective efficiency of the gratings under TE and TM polarization. From TE and TM efficiency measurements, we calculated the polarization sensitivity, defined as

$$
\left|\eta_{T E}-\eta_{T M}\right| /\left(\eta_{T E}+\eta_{T M}\right)
$$

where $\eta_{T E}$ and $\eta_{T M}$ correspond to efficiencies measured under TE and TM polarized light.

It results that the polarization sensitivity is smaller than $2 \%$ for both gratings over the whole spectral band, which confirms experimentally subwavelength optics polarization property.

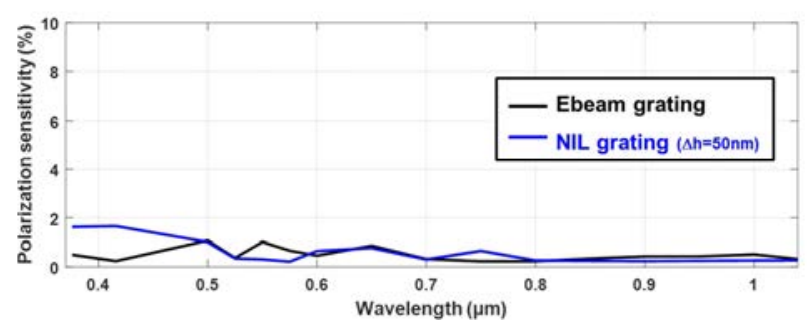

Fig. 6. Polarization sensitivity of the blazed-binary subwavelength gratings.

E-beam technology is well-known for its ability to achieve low level grating ghosts [11]. To verify this property for the NIL grating, we carried out angular scanning of many diffraction orders in the diffraction plane and plot the efficiency (power/reference) on the $y$ axis as a function of the detector angle on the $\mathrm{x}$-axis. The diffracted orders are separated by $1.3^{\circ}$ and the $-1^{\text {st }}$ order is located at $=-6.3^{\circ}$. It results that no additional peaks visible, meaning that no grating ghosts were measured. Note that this result is not surprising as the NIL technology uses a master based on e-beam lithography. However, it shows that the NIL technology doesn't introduce additional ghosts.

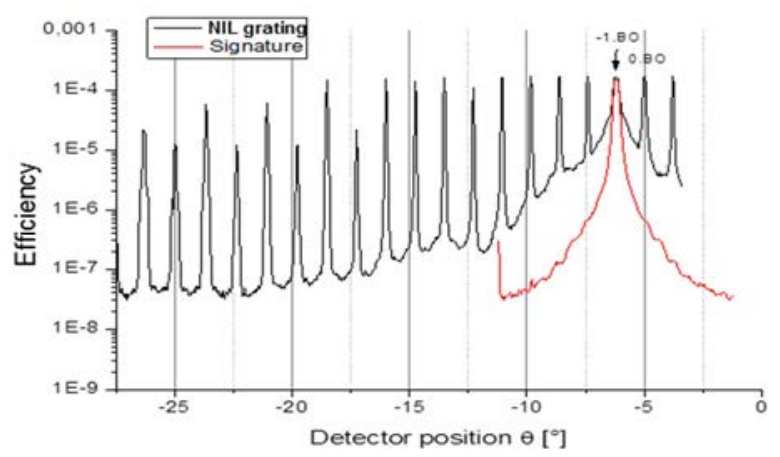

Fig. 7. Angular scanning of the efficiency in the diffractive plane at $633 \mathrm{~nm}$ for the NIL grating.

The wavefront error (WFE) introduced by the fabrication process is evaluated by measuring the reflected WFE of the gratings, using a plane-wave Zygo Verifire AT 12" interferometer at a wavelength of $633 \mathrm{~nm}$.

The wavefront deformation introduced by the fabrication process is evaluated as follows: 1) The retro-reflected wavefront of the grating is measured for collimated illumination obeying Littrow condition. A first measurement is performed in $+1^{\text {st }}$ diffraction order and a second measurement is independently performed in $-1^{\text {st }}$ diffraction order. 2) Both wavefront maps are then 
subtracted. This allows removing the influence of the grating substrate's deformation. The result only covers the inaccuracy introduced by the respective patterning process, see Fig. 8.
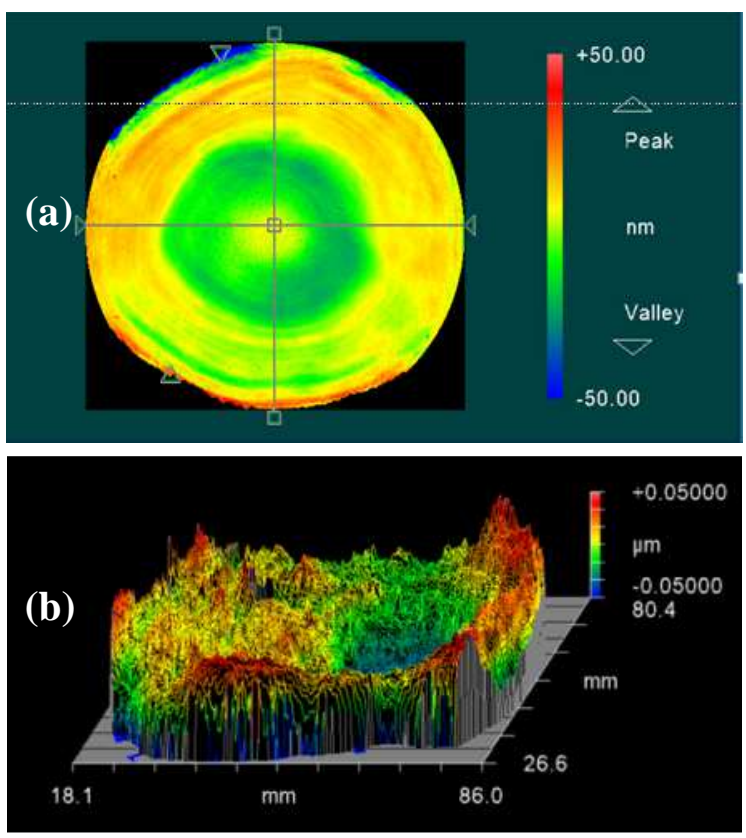

Fig. 8. Reflected wavefront introduced by the grating fabrication process (excluding substrate deformation) for e-beam grating (a) and for NIL grating (b).

For both technologies, the WFE is smaller than $20 \mathrm{~nm}$ RMS which is 5 times smaller than the $100 \mathrm{~nm}$ RMS space application requirement. Such results have already been demonstrated for e-beam technology [12], but it is striking that although NIL technology is less mature, requests additional process steps and is based on soft PDMS mold, its WFE over 3" size component is also excellent.

\section{Conclusion}

Starting from one single design, we have manufactured large area 3" subwavelength reflective gratings by two technologies (e-beam lithography and NIL). They are composed of high aspect ratio $(\sim 5)$, high density and one level subwavelength binary structures.

Optical measurements show that the subwavelength blazed-binary gratings technologies based on e-beam lithography or NIL are promising for space application: they enable superior performance, in term of spectral band (efficiency margin of $10-20 \%$ in average), polarization sensitivity (smaller than $2 \%$ ), very low ghost level (not detectable) and WFE level $(<20 \mathrm{~nm}$ RMS) with respect to requirements for observation imaging spectrometer operating in UV/Vis/NIR spectrometer requirements.
Part of this work was carried out within the framework of the ESA TRP project "Wide band and high efficiency reflective grating", No 4000118037/16/NL/PS.

\section{References}

1. N. Tetaz, C. Ruilier, M. Taccola, T. Viard, M.-S.L. Lee Bouhours, B. Loiseaux, G. Lehoucq, "Advanced large FOV UV/VIS/NIR/SWIR spectrometers for future earth observation instruments", Proc. SPIE 10563, 1056333 (2017).

2. S. M. Ebstein, " Achromatic diffractive optical elements", SPIE Vol. 2404, p211-216 (2001).

3. B. H. Kleemann, M. Seesselberg, and J. Ruoff, "Design concepts for broadband high-efficiency DOEs", J. Eur. Opt. Soc. 3, 08015 (2008).

4. O. Sandfuchs, M. Kraus, R. Brunner, "Structured metal double-blazed dispersion grating for broadband spectral efficiency achromatization", J. Opt. Soc. Am. A 37, 1369-1380 (2020).

5. P. Mouroulis, S.W. Wilson, P.D. Maker, R.E. Muller, "Convex grating types for concentric imaging spectrometers", Appl. Opt. 37(31), p72007208 (1998).

6. B. Van Gorp, P. Mouroulis, D. W. Wilson, R. O. Green, "Design of the Compact Wide Swath Imaging Spectrometer (CWIS) ", Proc. SPIE 9222, Imaging Spectrometry XIX, 92220C (2014).

7. C. Sauvan, P. Lalanne, M-S. L. Lee, "Broadband blazing with artificial dielectrics", Opt. Lett. 29, 1593-1595 (2004).

8. C. Ribot, M.-S.L. Lee, S. Collin, S. Bansropun, P. Plouhinec, D. Thenot, S. Cassette, B. Loiseaux, P. Lalanne, "Broadband and Efficient Diffraction", Adv. Opt. Mat. 1(7), p489-493 (2013).

9. F. Burmeister, T. Flügel-Paul, U.D. Zeitner, M.-S. L. Lee-Bouhours, G. Lehoucq, J. Cholet, B. Loiseaux, N. Tetaz, R. Windpassinger, M. Taccola, "Binary blazed reflection grating for UV/VIS/NIR/SWIR spectral range", Proc. SPIE 11180, ICSO 2018, 111801J (2019).

10. W. Stork, N. Streibl, H. Haidner, and P. Kipfer, "Artificial distributed-index media fabricated by zero-order gratings", Opt. Let. 16(24), pp. 19211923 (1991).

11. M. Heusinger, M. Banasch, T. Flügel-Paul, U. D. Zeitner, "Investigation and optimization of Rowland ghosts in high efficiency spectrometer gratings fabricated by e-beam lithography", Proc. SPIE 9759, Advanced Fabrication Technologies for Micro/Nano Optics and Photonics IX, 97590A (2016).

12. M. Erdmann, E.-B. Kley, U. Zeitner, "Development of a large blazed transmission grating by effective binary index modulation for the GAIA radial velocity spectrometer", Proc. SPIE 105650, ICSO 2010, 105651N (2017). 